\title{
Second-Order Kalman Filtering Application to Fading Channels Supported by Real Data
}

\author{
Azra Kapetanovic, Redhwan Mawari, Mohamed A. Zohdy \\ Department of Electrical and Computer Engineering, Oakland University, Rochester, MI, USA \\ Email: akapetan@oakland.edu,rmawari@oakaland.edu,zohdyma@oakland.edu
}

Received 9 February 2016; accepted 9 May 2016; published 12 May 2016

Copyright (C) 2016 by authors and Scientific Research Publishing Inc.

This work is licensed under the Creative Commons Attribution International License (CC BY). http://creativecommons.org/licenses/by/4.0/

(c) () Open Access

\begin{abstract}
The lack of effective techniques for estimation of shadow power in fading mobile wireless communication channels motivated the use of Kalman Filtering as an effective alternative. In this paper, linear second-order state space Kalman Filtering is further investigated and tested for applicability. This is important to optimize estimates of received power signals to improve control of handoffs. Simulation models were used extensively in the initial stage of this research to validate the proposed theory. Recently, we managed to further confirm validation of the concept through experiments supported by data from real scenarios. Our results have shown that the linear second-order state space Kalman Filter (KF) can be more accurate in predicting local shadow power profiles than the first-order Kalman Filter, even in channels with imposed non-Gaussian measurement noise.
\end{abstract}

\section{Keywords}

Kalman Filtering, Rayleigh, Gaussian, Multipath, Shadowing, Power Estimation

\section{Introduction}

In the fast growing field of wireless communication technology, accurate estimation of local mean shadow power at a mobile station (MS) is becoming a popular area of challenge for engineers in both industry and academia. There is an ever present need to continue to find ways to enhance device performance in power control and handoff, particularly to address mobility-induced fading.

Due to the motion of the mobile station (i.e. cellphone), the received signal strength fluctuates with two multiplicative forms of fading, shadowing (local mean, local power) and multipath [1]. Shadowing is power loss in the communication signal caused by the presence of large obstructions in the path of the moving station and the base station (BS) [2]. For example, in the outdoor environment, shadow fading in cellphones causes long-term variation primarily caused by nearby mountains or tall buildings. Figure 1(a) illustrates random variation in

How to cite this paper: Kapetanovic, A., Mawari, R. and Zohdy, M.A. (2016) Second-Order Kalman Filtering Application to Fading Channels Supported by Real Data. Journal of Signal and Information Processing, 7, 61-74.

http://dx.doi.org/10.4236/jsip.2016.72008 
path loss as the cellphone user moves around a building while using the cell phone. Tall building structure shadows the radio signal, which results in power drop at a receiver input. Multipath, another fading phenomenon in mobile communications, results in fluctuations of the signal amplitude because of the addition of signals that arrive with different phases [3]. This is due to the fact that transmitted signals travel different distances by along different paths as illustrated in Figure 1(b). When comparing these two forms of fading, it is important to note that the multipath signal component has a much higher frequency than shadowing. Many common shadow power estimation methods in industry rely on an accurate model for multipath. Multipath is often modeled as Rayleigh Noise for modeling purposes [4].

Mobile communication shadowing plays a huge role in handoff decisions and power control. In [6], handoff is defined as the process of transferring an active call from one cellphone in a cellular network to another. A well-implemented handoff is important for delivering uninterrupted service to a caller. Therefore, having accurate estimation of the shadowing component of a received power signal can help the system perform handoff at the most effective times (predict when and where to handoff user). In order to achieve an acceptable signal quality, the path loss from the shadowing component of a received power signal has to be compensated by the transmitter.

Having an accurate estimate of shadowing will allow the mobile communication system to efficiently compensate for the signal degradation that will occur. In industry, several different types of windows-based estimators are utilized to filter multipath noise from the instantaneous received power signal in order to estimate the local mean shadow power. These windows-based estimators work well under the assumption that the shadowing component is relatively constant during the window period. In [1], we learn that the shadowing components do change slowly, but the fluctuations can vary and at times can significantly decrease the performance of the windows-based estimate. The need for a method of determining the optimal window period is clear; however, present day research has not revealed a consistent closed form solution that takes into account all system and propagation parameters of shadowing.

The lack of a consistent technique for calculating an optimized window period provides the motivation for finding an alternative method to windows-based estimation for estimating local mean shadow power. In our research, goal was to investigate an alternative method called Kalman Filtering. Kalman Filtering, introduced in [6], is also known as best linear unbiased estimation, is very effective when measurement noises are assumed Gaussian and the channel is linear fading, also with Gaussian noise. The Kalman algorithm uses a series of measured observations and produces optimal estimates of statesas explained in [7] [8].

In this paper, our contribution is to study and analyze output results of the first-order state space and to compare them to the second-order state space models while applying a Kalman Filter technique to determine shadow power signal in mobile communications from measurements that have impinged Rayleigh fast fading noise [9]. We propose using a second-order state space Kalman Filter estimator to estimate local mean (shadow) power. In this paper, we demonstrate that thesecond-order KF exceeds the performance of the first-order KF-based estimator. We produced new results and validated this concept by conducting experiments with real scenario data. We also propose a new yet unvalidated adaptive handoff based on a balance of shadow signal strength and quality.

\section{Shadow Power Signal}

A model for instantaneous received power $l(t)$ at a Mobile Station (MB) in a wireless communication is given in Figure 2, where $|w(t)|^{2}$ represents fast power fluctuation due to multipath and $s(t)$ represents slow power

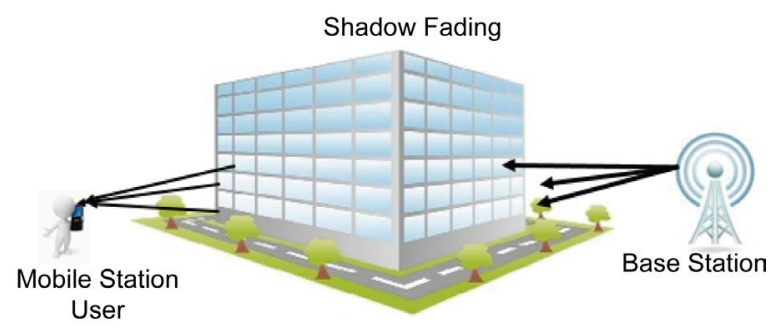

(a)

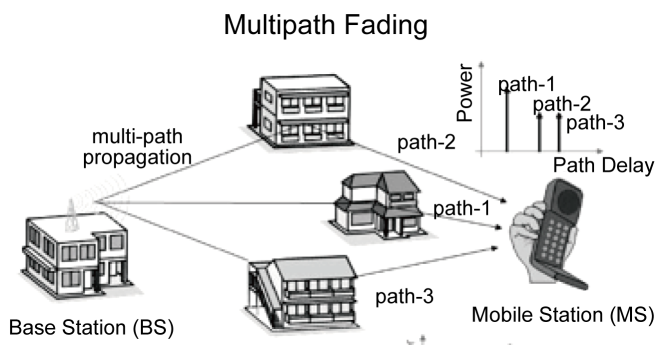

(b)

Figure 1. (a) The shadow fading, and (b) the multiple transmission paths at the receiver [5]. 


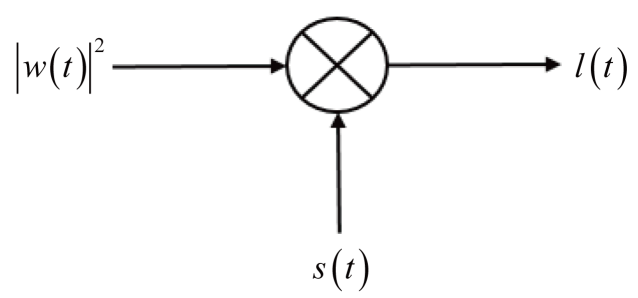

Figure 2. Model for received power.

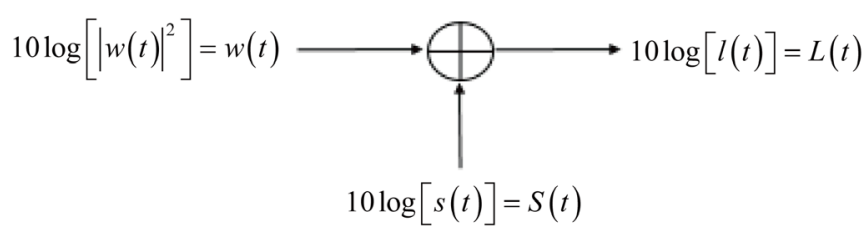

Figure 3. Applying log function.

fluctuation due to shadowing. It is customary to express power measurements in decibels as shown in Figure 3.

To solve this problem, we start with the multipath model shown in Equation (2),

$$
\begin{array}{r}
\square w(R)=\frac{1}{R}\left[a_{1} \mathrm{e}^{j\left(\omega_{D} \cos \left(\theta_{1}\right) t+\phi_{1}\right)}+a_{2} \mathrm{e}^{j\left(\omega_{D} \cos \left(\theta_{2}\right) t+\phi_{2}\right)}+a_{R} \mathrm{e}^{j\left(\omega_{D} \cos \left(\theta_{R}\right) t+\phi_{R}\right)}\right] \\
w(R+1)=\frac{1}{R+1}\left[w(R) * R+a_{R+1} \mathrm{e}^{j\left(\omega_{D} \cos \left(\theta_{R+1}\right) t+\phi_{R+1}\right)}\right]
\end{array}
$$

where

$\bullet v$ is the magnitude of the mobile velocity $\left[10 \frac{\mathrm{m}}{\mathrm{s}}-30 \frac{\mathrm{m}}{\mathrm{s}}\right]$,

- $\lambda$ is the wavelength corresponding to the carrier frequency, which is typically $\frac{3 \times 10^{8}}{700 \mathrm{MHz}}=0.42 \mathrm{~Hz}$,

- $\omega_{D}$ is Doppler spread equal to $\frac{2 \pi v}{\lambda}$. (Range used in this paper is $\left[\frac{2 \times \pi \times 10 \frac{\mathrm{m}}{\mathrm{s}}}{\frac{3}{7} \mathrm{~Hz}}-\frac{2 \times \pi \times 30 \frac{\mathrm{m}}{\mathrm{s}}}{\frac{3}{7} \mathrm{~Hz}}\right]$ ),

- $R$ is the number of paths in multipath power [20 - 30],

- $a_{r}$ is gain [0 - 20],

- $\theta_{r}$ represents angles between incoming waves and mobile antenna. Value range is uniformly distributed $[-\pi, \pi]$. Other distributions like Normal can be used.

- $\left\{\phi_{r}\right\}_{r=1}^{R}$ represents phase random variables whose values are also uniformly distributed $[-\pi, \pi]$. (We may consider other random distributions.)

\section{Linear Kalman Filter Algorithm}

The Kalman Filter is a form of a linear algorithm for optimal recursive estimation of a system state with a specific set of output equations (Figure 4). This algorithm was used to generate the first part of a MATALB code during the initial state of the research. References [10] and [11] were useful for programing in MATLAB during initial stage of research.

To build an estimation model in MATLAB, we started with Equations (3) and (4), with suitable entries to reflect the linear channel model [12]. This assumes alinear time invariant system with zero mean and white noise on both the state and output.

$$
\begin{gathered}
x_{k}=A x_{k-1}+B u_{k}+w_{k-1} \\
l_{k}=H x_{k}+v_{k}
\end{gathered}
$$

where 
- $x_{k}$ is a symbol value of shadow signal state that needs to be estimated,

- $u_{k}$ is the control signal for handoff,

- $w_{k-1}$ is process white noise,

- $v_{k}$ is measurement noise,

- $l_{k}$ is the measurement value for both shadow and multipath. In this paper, $l_{k}$ or $L(k)$ is the measurement value used to update our shadow power estimate.

Assume initial values to be $x_{O}$ and $P_{O}$. For ease of reference, a list of the principal symbols used in given below:

$K_{k}$ is Kalman gain,

$R$ is environment noise covariance,

$Q$ is system noise covariance,

$P$ is error covariance.

Prediction States:

Equations (5) and (6) represent the "Time Update" state of the Kalman Filter, also known as "Prediction States." Here, we project the current state estimate ahead (in time). Equation (5) projects the state ahead and equation (6) projects the error covariance ahead. $K_{k}$ Denotes Kalman gain,

$$
\begin{gathered}
\hat{x}_{k}^{-}=A \hat{x}_{k-1}+B u_{k} \\
P_{k}^{-}=A P_{k-1} A^{\mathrm{T}}+Q
\end{gathered}
$$

$\hat{x}_{k}^{-}$is the rough estimate before the measurement update correction.

Correction States:

Equations (7), (8) and (9) belong to the "Measurement Update" state of the Kalman Filter, also known as the "Correction State." Here, we adjust the projected estimate by an actual measurement. Equation (7) computes the Kalman Gain, Equation (8) adjusts the projected estimate by an actual measurement $l_{k}$, and Equation (9) updates the error covariance.

$$
K_{k}=P_{k}^{-} H^{\mathrm{T}}\left(H P_{k}^{-} H^{\mathrm{T}}+R\right)^{-1}
$$

If $R$ is small and $P_{k}^{-}$is close to Identity, then $K_{k}=H^{\mathrm{T}}\left(H H^{\mathrm{T}}\right)^{-1}$, which is a well-known Pseudo inverse. $\hat{x}_{k}$ : Estimate of $x$ at time $k$.

$$
\begin{gathered}
\hat{x}_{k}=\hat{x}_{k}^{-}+K_{k}\left(l_{k}-H \hat{x}_{k}^{-}\right) \\
P_{k}=\left(I-K_{k} H\right) P_{k}^{-}
\end{gathered}
$$

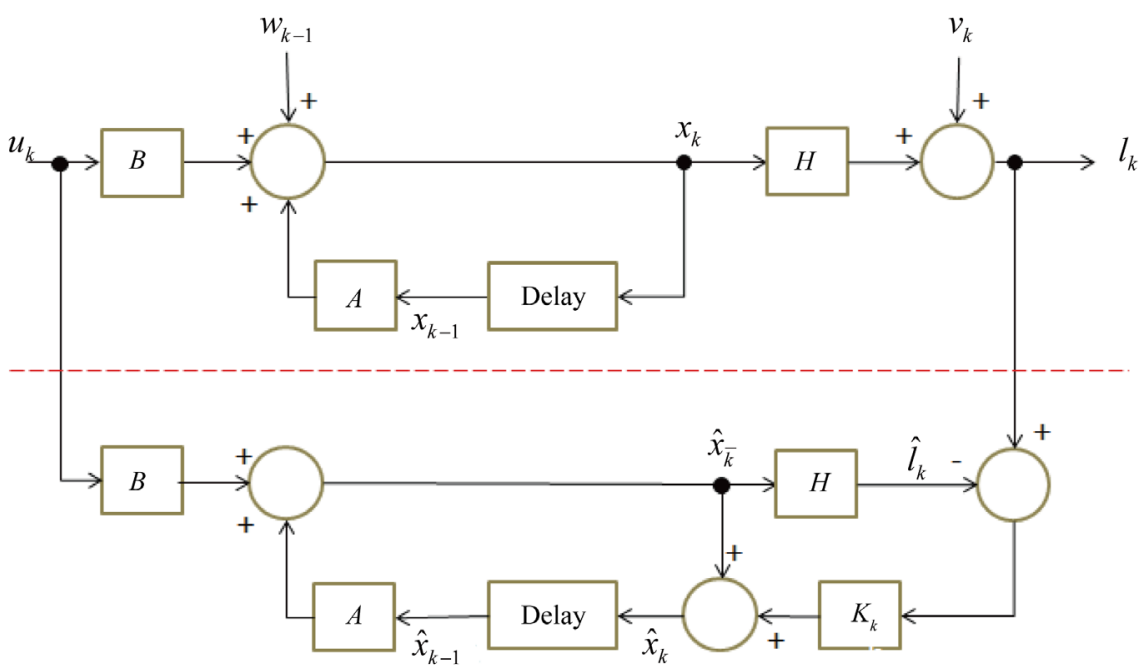

Figure 4. A block diagram for Kalman Filter (http://www.swarthmore.edu/NatSci/echeeve1/Ref/Kalman/MatrixKalman.html). 
The next step is to iterate through the estimates for a sufficient time. The output estimate in the previous step will be the input estimate in the next step. The main goal is to find an optimal value for $\hat{x}_{k}$.

\section{Second-Order State Space for Linear Kalman Filter}

In this research, we assumed that the first-order order state space model can be used to model the Shadow Power.

To extend the first-order state space model equations [1] introduced in Section 3, a second-order state space model for the linear Kalman Filter was formulated and applied as suggested in the following equations.

$a_{1}$ is the first Shadow power coefficient.

$a_{2}$ is the second Shadow power coefficient.

$X_{c}$ is the effective correlation distance [10 - $\left.500 \mathrm{~m}\right]$.

$\varepsilon_{D}$ is the correlation coefficient of shadow process between two points separated by distance $D$ measured in meters.

$$
\begin{gathered}
X_{c}=\frac{-D}{\ln \left(\varepsilon_{D}\right)} \\
a_{1}=\mathrm{e}^{\frac{-v T_{s}}{X_{c 1}}} \\
a_{2}=\mathrm{e}^{\frac{-v T_{s}}{X_{c 2}}} \\
x_{k}=\left[\begin{array}{c}
X_{1 k} \\
X_{2 k}
\end{array}\right]
\end{gathered}
$$

Prediction States for Second-order Linear KF:

Equation (5) can be rewritten as Equation (14). Therefore, equation (14) in this section projects the state ahead, and equation (16) projects the error covariance ahead.

For second-order, matrices are defined as follows:

$$
\begin{gathered}
A=\left[\begin{array}{cc}
1 & 0 \\
a_{1 k} & a_{2 k}
\end{array}\right], \quad B=\left[\begin{array}{l}
0 \\
0
\end{array}\right], \quad H=\left[\begin{array}{ll}
0 & 1
\end{array}\right] \\
{\left[\begin{array}{c}
\hat{x}_{1 k}^{-} \\
\hat{x}_{2 k}^{-}
\end{array}\right]=\left[\begin{array}{cc}
1 & 0 \\
a_{1 k} & a_{2 k}
\end{array}\right]\left[\begin{array}{l}
\hat{x}_{1 k-1} \\
\hat{x}_{2 k-2}
\end{array}\right]}
\end{gathered}
$$

Parameter $Q$ in Equation (16) represents the predicted process noise as shown in Equation (15). $\sigma_{s}$ : denotes the shadow variance $[4 \mathrm{~dB}-8 \mathrm{~dB}]$.

$\left(1-a^{2}\right) \times \sigma_{s}^{2}$ is variance of the zero mean white Gaussian noise.

$$
Q=\left[\begin{array}{cc}
\left(1-a_{1}^{2}\right) \sigma_{s}^{2} & \left(1-a_{2} a_{1}\right) \sigma_{s}^{2} \\
\left(1-a_{2} a_{1}\right) \sigma_{s}^{2} & \left(1-a_{2}^{2}\right) \sigma_{s}^{2}
\end{array}\right]
$$

Equation (6) can be then expressed in the following state space format:

$$
P_{k}^{-}=\left[\begin{array}{cc}
1 & 0 \\
a_{1 k} & a_{2 k}
\end{array}\right] P_{k-1}\left[\begin{array}{cc}
1 & a_{1 k} \\
0 & a_{2 k}
\end{array}\right]+Q
$$

Correction States for Second-order Linear KF:

Equation (17) was used to compute Kalman gain, where $R$ represents the measurement noise due to multipath.

$$
\begin{gathered}
H=\left[\begin{array}{ll}
0 & 1
\end{array}\right] \\
H^{\mathrm{T}}=\left[\begin{array}{l}
0 \\
1
\end{array}\right]
\end{gathered}
$$




$$
K_{k}=P_{k}^{-} H^{\mathrm{T}}\left(H P_{k}^{-} H^{\mathrm{T}} R\right)^{-1}
$$

Equation (18) updates the estimate via $l_{k}$, a measured value, and Equation (19) updates the error covariance.

$$
\begin{gathered}
{\left[\begin{array}{c}
\hat{x}_{1 k} \\
\hat{x}_{2 k}
\end{array}\right]=\left[\begin{array}{l}
\hat{x}_{1 k}^{-} \\
\hat{x}_{2 k}^{-}
\end{array}\right]+K_{k}\left(l_{k}-H\left[\begin{array}{l}
\hat{x}_{1 k}^{-} \\
\hat{x}_{2 k}^{-}
\end{array}\right]\right)} \\
P_{k}=\left(I-K_{k} H\right) P_{k}^{-}
\end{gathered}
$$

$X^{*}$ in Equation (20) is the optimal estimate of the second order shadow process.

$$
X^{*}=H\left[\begin{array}{l}
\hat{x}_{1 k} \\
\hat{x}_{2 k}
\end{array}\right]
$$

When the channel is nonlinear, the Unscented Kalman Filter also can be applied to the state space model to further improve and optimize the shadow power presented in this section. We may consider this optimization in the future.

\section{Handoff Decisions}

In wireless cellular communications, accurate local mean (shadow) power estimation performed at a mobile station is important for use in power control, handoff and adaptive transmission [1]. In [13], the term handoff or handover refers to the process of transferring an ongoing message or data from one channel connected to a base station to another base station. Handoffs are classified into two categories, soft handoff and hard handoff, as depicted in Figure 5 and Figure 6.

The term soft handoff in mobile communications refers to the process of transferring a phone call currently in progress from one base station to another base station without any interruptions to the call. Figure 5 illustrates this type of handoff and shows that there is a big overlap in signals coming from two different base stations. This process is also referred to as make-before-break, and it is unique to cell phones that use the Code Division Multiple Access radio system.

The term hard handoff refers to the process of transferring a phone call where connection to one base station (named source cell) is broken before the connection to another base station (named target cell) is established.

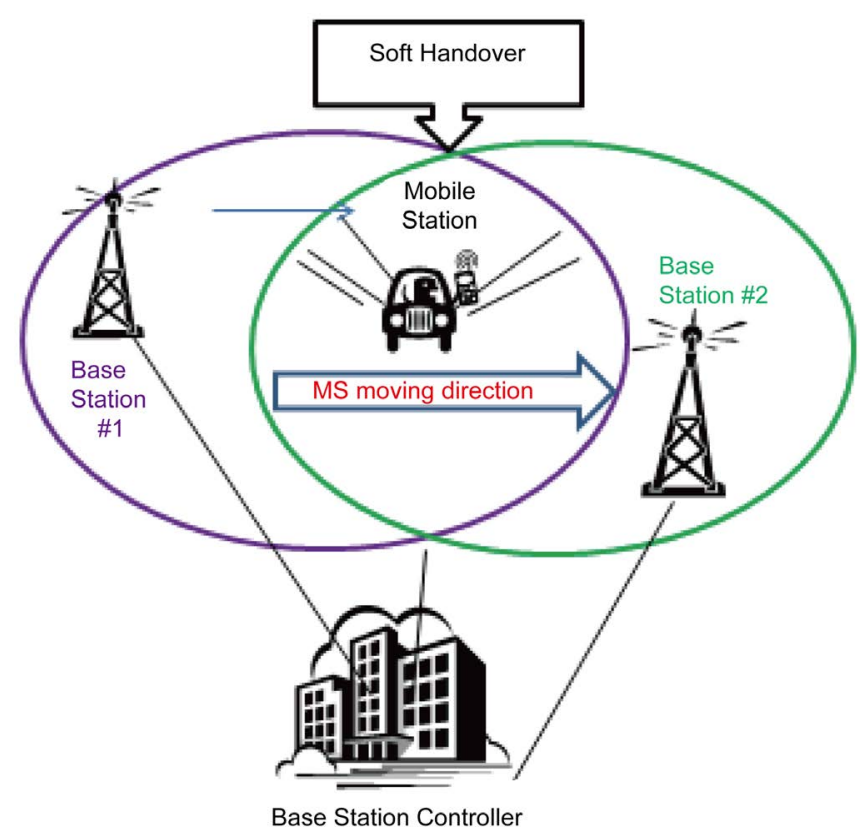

Figure 5. Example of soft handoff process (big overlap). 


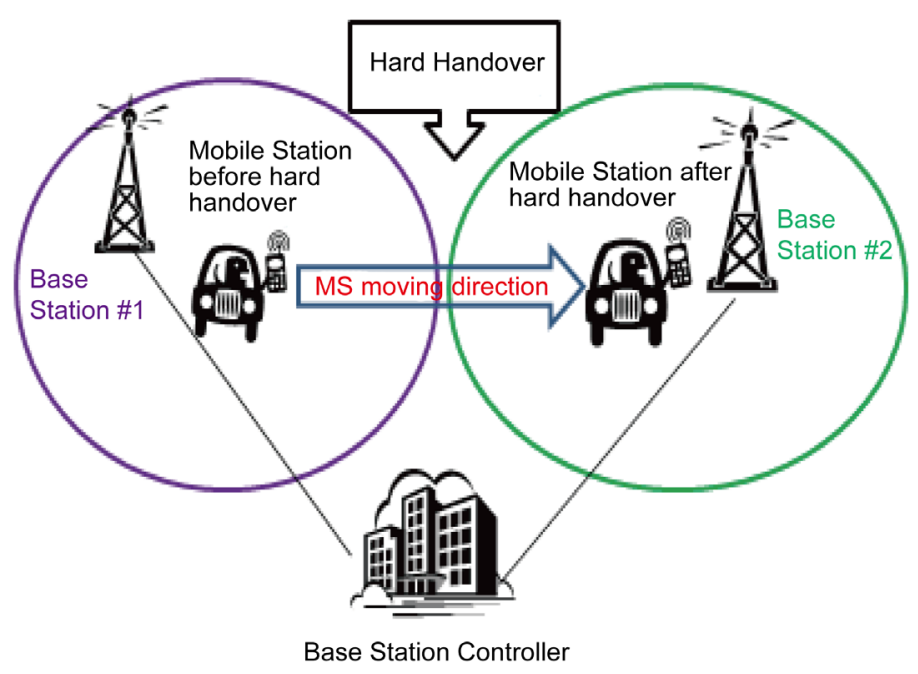

Figure 6. Example of hard handoff process (no overlap).

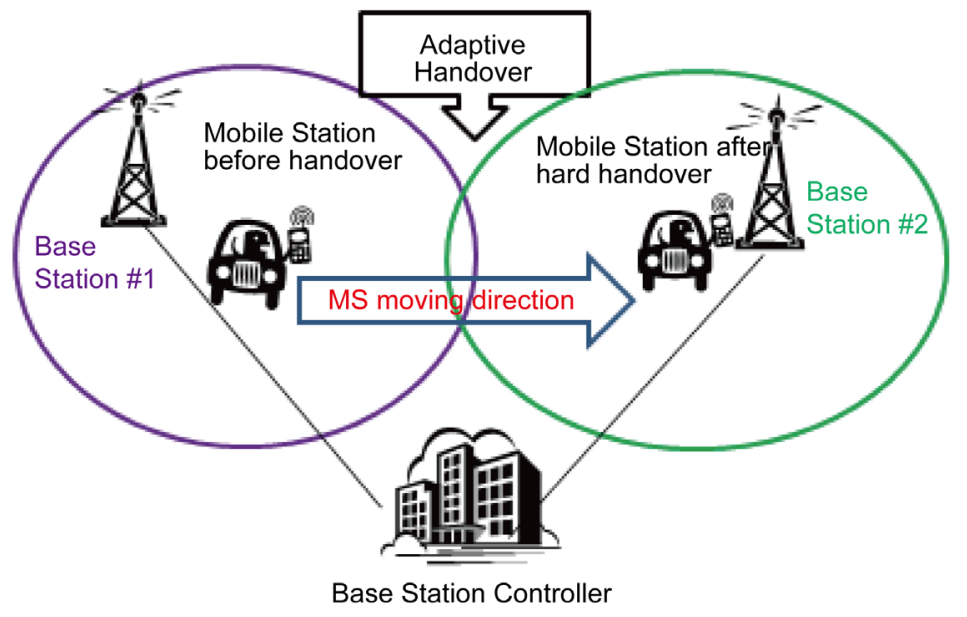

Figure 7. Example of adaptive handoff process (small overlap).

Thus, an alternative name for this process is break-before-make and it is unique to cell phones that operate using the Global System for Mobile Communications. As depicted in Figure 6, there is no overlap in the hard handover process. The interval during which the hard handoff takes place, should be extremely short to minimize disruption to the call. Both hard handoff and soft handoff events should not be noticeable by the user, but a soft handoff is generally more reliable.

We propose a new adaptive handoff based on predictive function of the estimated part. Figure 7 illustrates this type of handoff and shows that there is a small overlap in signals coming from two different base stations.

It is important to note that the land area supplied with radio service is divided into hexagonal cells. Each cell operates on a group of designated frequencies $\left(f_{1}, f_{2}, f_{3}, f_{4}, f_{5}, f_{6}\right)$. The same group of frequencies cannot be used in an adjacent neighboring hexagonal cell, but it can be used in other nearby locations. See the illustration in Figure 8 [14].

\section{Results for the Second-Order Linear Kalman Filter}

Previous reviewers on an earlier version of the manuscript suggested that we produce new results for estimation of local mean power by using the second-order state space Kalman Filter. Section 5.1 briefly explains Lab Setup used to conduct experiments and collect real data. Section 5.2 shows that we successfully validated our concept by using computer simulation. Section 5.3 presents results that show how we implemented idea in real scenarios where the fading model is unknown. 


\subsection{Experimental Setup}

Current power measurement techniques are only able to provide a limited number of measurement schemes, which are not enough to asses such a complex phenomenon. There had been a great consideration and search in the area of measurements and modeling of the propagation effects in the indoor environments. However, such work only considered a specific environment explicitly stationary scenarios as reported in [15] [16], and thus the empirical results and modeling cannot represent a mobile device environment, where the wireless communication takes place while the mobile device is moving. In December of 2015, authors of [17] presented an alternative measurement technique that enabled us to conduct experiment where wireless devices are moving in more than one direction.

As part of this research, we were able to setup and conduct good experiments by utilizing an Electrical Engineering Laboratory at Georgia Tech University, shown in Figure 9(a). This Laboratory is approximately $7 \mathrm{~m}$ long and $4 \mathrm{~m}$ wide. The environment inside the lab had various obstacles between the transmitter and receiver (e.g. metal shelves, tables, chairs, and testing lab equipment located against the walls). The purpose of the experiment was to obtain the received power signal. Objects obstructing the propagation path between the transmitter and receiver can cause variations in the received signal.

Figure 10 shows that the two components are required for communication: a transmitter and a receiver.

\section{Stationary Transmitter:}

Signal generator: Generates a $2.43 \mathrm{GHz}$ signal in order to transmit the signal at power levels- $5 \mathrm{dBm}$ and 15 $\mathrm{dBm}$.

Omnidirectional transmitter antenna: Converts generated signal to produce radio waves.

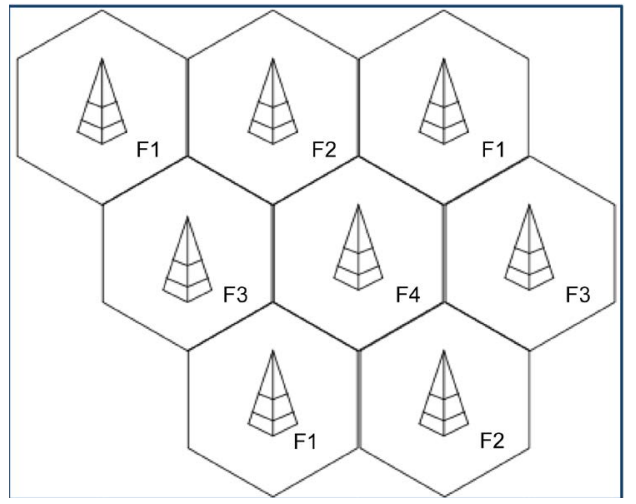

(a)

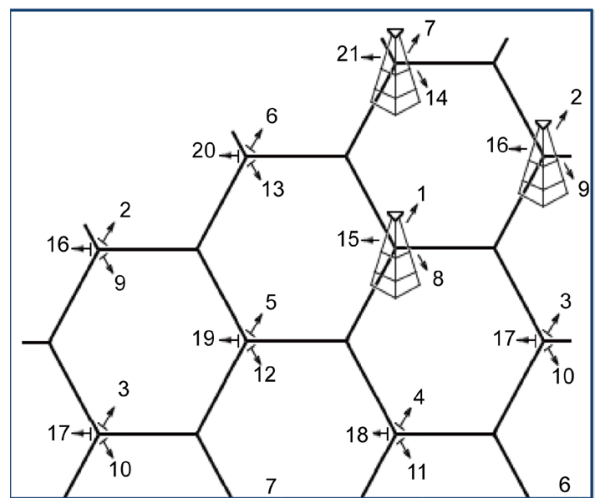

(b)

Figure 8. (a) Hexagonal shape cells, and (b) the designated frequency sets [14].

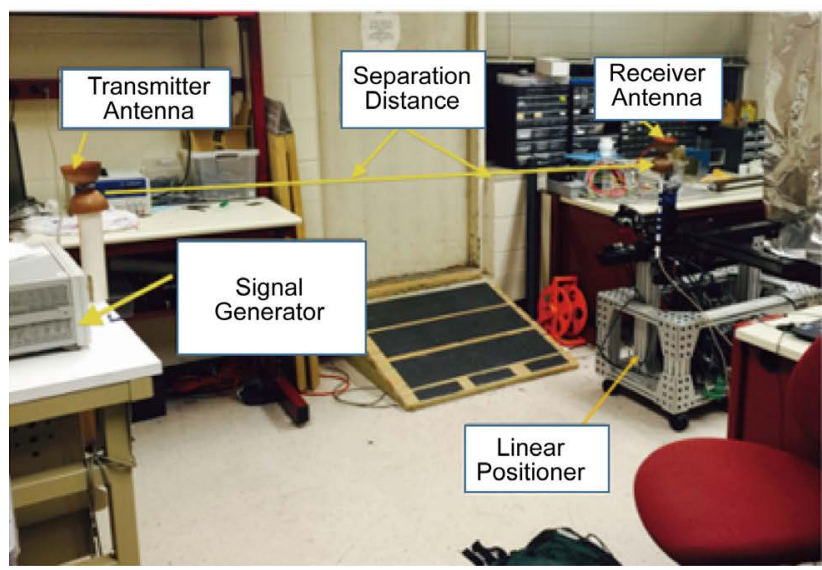

(a)



(b)

Figure 9. (a) Lab setup used to collect real data, and (b) the linear positioner machine. 


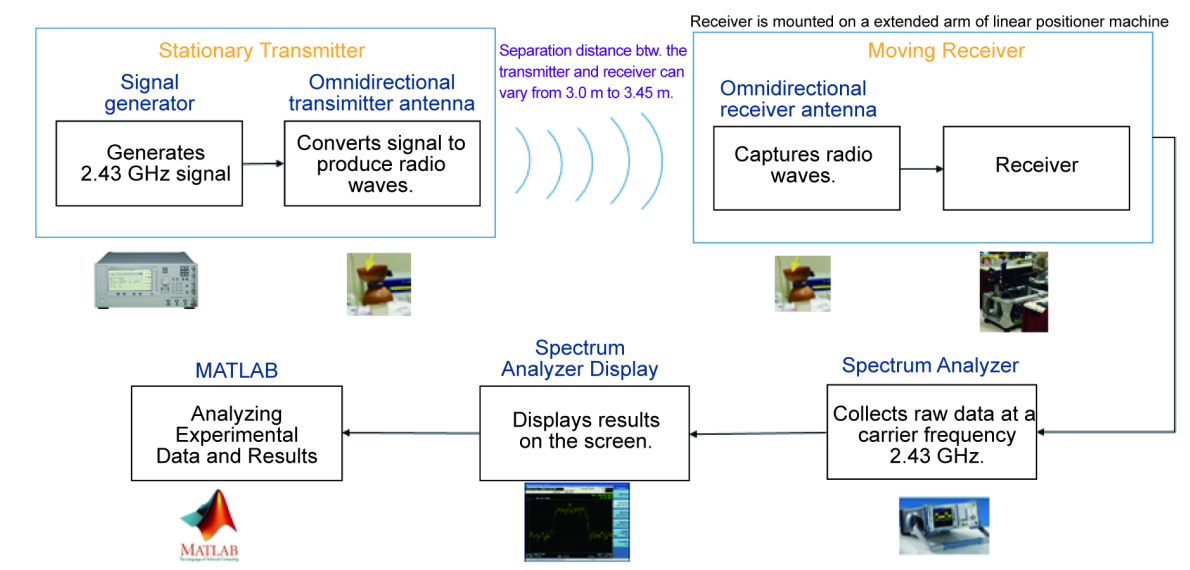

Figure 10. Block diagram of wireless communication system used in the lab environment.

\section{Moving Receiver:}

Omnidirectional receiver antenna: Captures the radio waves to receive the signal.

Receiver: The receiver is mounted on an extended arm of the linear positioner machine, which moves toward and away from the transmitter along the $\mathrm{x}$-axis and $\mathrm{y}$-axis. Therefore, in this experiment, the separation distance between the transmitter and receiver varied from $3 \mathrm{~m}$ to $3.45 \mathrm{~m}$. The extended arm moves in a two-dimensional manner: $45.7 \mathrm{~cm}$ along the $\mathrm{x}$-axis and $45.7 \mathrm{~cm}$ along the $\mathrm{y}$-axis, with $2.5 \mathrm{~cm}$ step size (see Figure 9(b)).

\section{Additional Tools:}

Spectrum analyzer: Collects the raw data (i.e., the received power level at a given position in $\mathrm{x}$ and $\mathrm{y}$ directions) and loads it into the computer.

Spectrum analyzer display: Displays results on the screen.

MATLAB Software: Reads the raw data from the spectrum analyzer. It was also used to load data into second-order Kalman Filter MATLAB Code for analysis purposes.

\subsection{Simulation Results}

This section presents computer simulation results. Simulation models were created and used extensively in the initial stage of this research to validate the proposed theory. Figure 13 shows MATLAB results for multipath power, shadow power and total power without Kalman Filtering. Figure 11 shows results of the actual shadow power signal and estimation with Kalman Filtering. Default conditions for base parameters are:

$\bullet v=10 \mathrm{~m} / \mathrm{s}$ (constant velocity).

- $R=20$ (number of paths in multipath power).

- $T_{s}=100 \mathrm{~ms}$ (sampling time).

- $X_{c}=200$ (same for both shadow power terms).

- $\sigma_{\mathrm{s}}=6 \mathrm{~dB}$ (shadow variance).

The second-order Kalman Filter shown in Figure 11 includes process state cross covariance. The graph line marked in green represents the signal without KF applied to it, and the line marked in red is the second-order Kalman Filter estimate. Authors in [1] show that first-order Kalman Filter method is superior to conventional window-based estimators like sample average estimator, the uniformly minimum variance unbiased estimator, and the maximum likelihood estimator. Our simulation results show that the second-order KF improves the signal estimate significantly over the first-order KF estimate.

Simulation was run to compare the system performance with the integrated second-order KF versus the same system with the first-order KF. Figure 12 indicates that the second-order KF tracks shadow power better than first-order KF. Average error for the second-order KF is lower than first-order KF. Table 1 provides a summary of MATLAB simulation trials with applied Kalman Filtering while varying the default parameters for mobile receiver velocity, shadow variance and effective correlation distance. For example, the mobile receiver velocities varied from $1 \mathrm{~m} / \mathrm{s}$ (walking) to $10 \mathrm{~m} / \mathrm{s}$ (city driving) or $30 \mathrm{~m} / \mathrm{s}$ (highway driving). The second-order Kalman Filter performed equally well when we varied the parameters listed in Table 1, which indicates the robustness of our concept. 


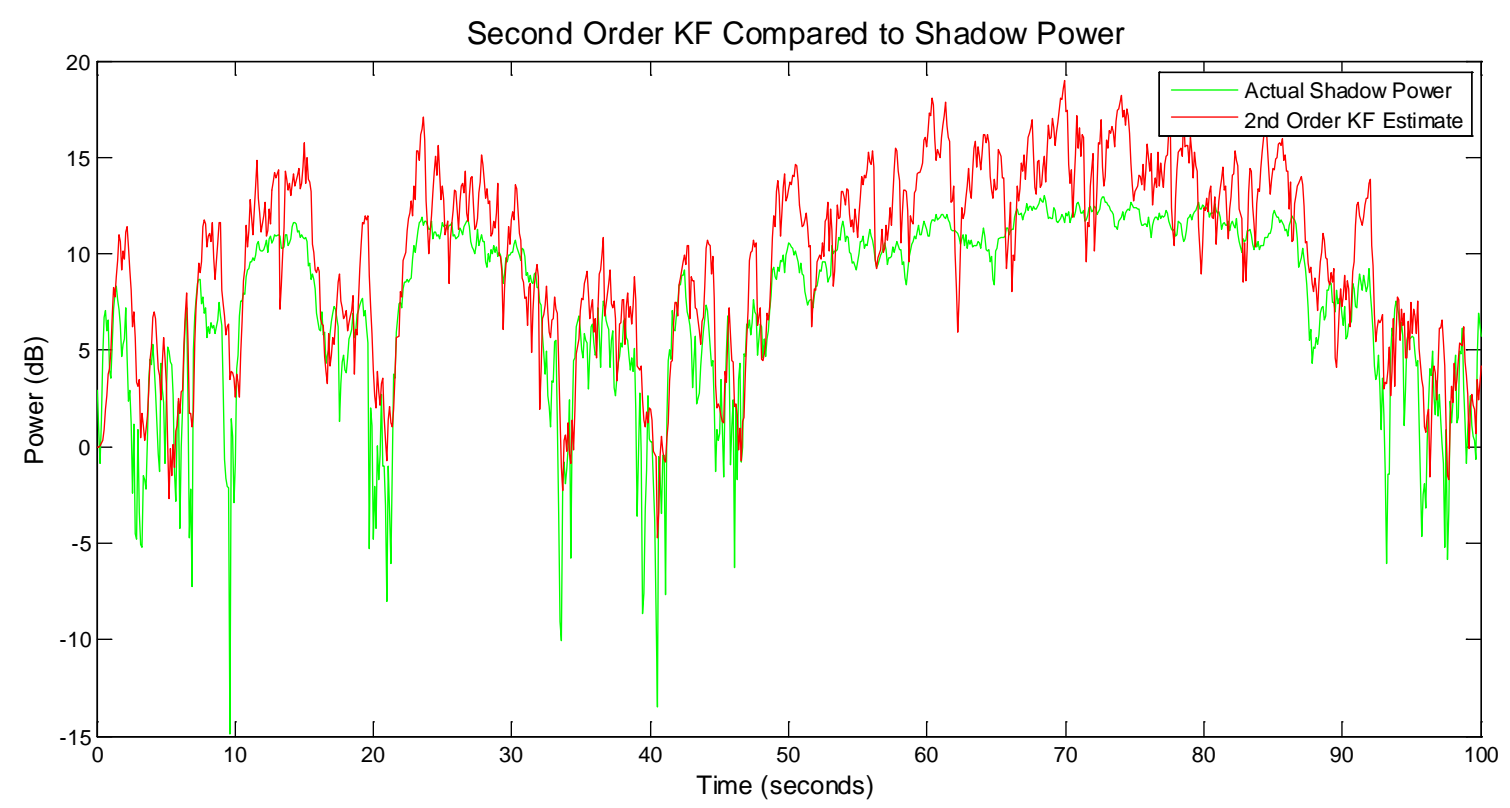

Figure 11. Estimation with second-order state space model with Kalman Filter.

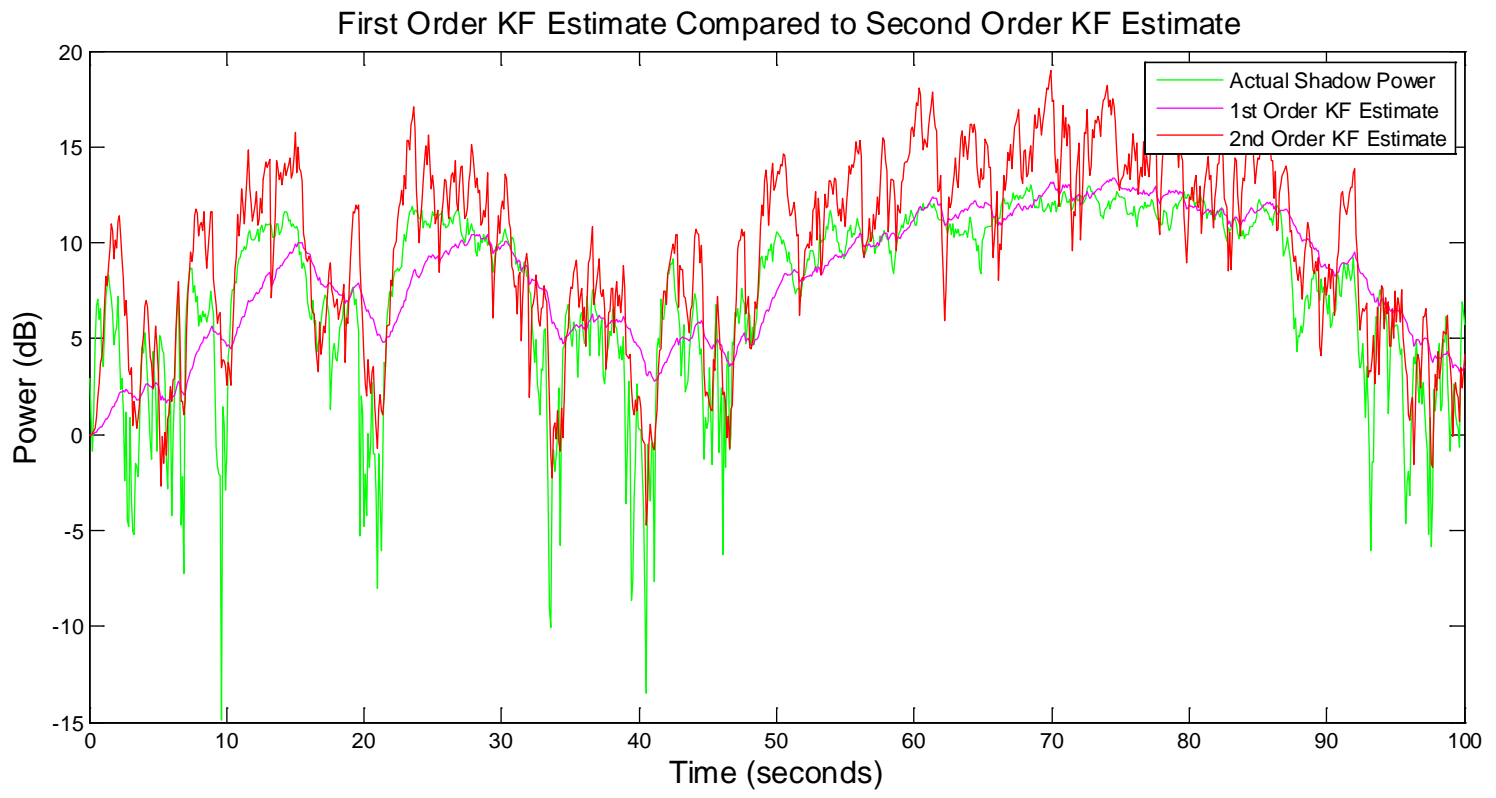

Figure 12. Comparison of first-order and second-order state estimates with Kalman Filter applied.

Table 1. Statistical performance for second-order KF estimation with varying parameters.

\begin{tabular}{cccccc}
\hline $\begin{array}{c}\text { Changing } \\
\text { parameter }\end{array}$ & Notation & $1^{\text {st }}$ test & $2^{\text {nd }}$ test & $3^{\text {rd }}$ test & Second-order KF performance \\
\hline $\begin{array}{c}\text { Mobile receiver velocity } \\
\text { Shadow variance }\end{array}$ & $(v)$ & $1 \mathrm{~m} / \mathrm{s}$ & $10 \mathrm{~m} / \mathrm{s}$ & $30 \mathrm{~m} / \mathrm{s}$ & $\begin{array}{c}\text { KF performed well when mobile receiver } \\
\text { velocity was varied. }\end{array}$ \\
$\begin{array}{c}\text { Effective correlation } \\
\text { distance }\end{array}$ & $\left(\sigma_{s}\right)$ & $4 \mathrm{~dB}$ & $6 \mathrm{~dB}$ & $8 \mathrm{~dB}$ & KF performed well when shadow variance was varied. \\
KF performed well when effective correlation \\
distance was varied.
\end{tabular}

Total power is the combination of multipath power and shadow power as illustrated in Figure 13. The graph line marked in blue in Figure 14 represents the total power signal. Graph line marked in pink illustrates 

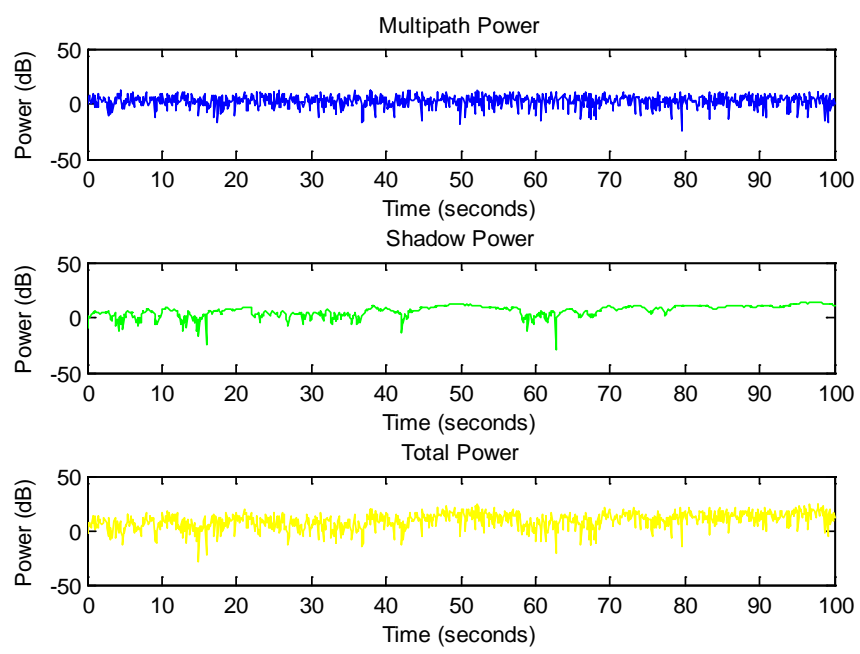

Figure 13. Second-order state space model.



Figure 14. Comparison of total power estimates.

performance of the first-order KF prediction for the total power. Similarly, the graph line marked in green illustrates performance of the second-order KF prediction for the total power. The results have been consistent in showing that the implementation of the second-order KF results in better estimation.

\subsection{Experiment Results Supported by Real Data}

A previous reviewer of an earlier version of this manuscript suggested completing validation for this concept through lab experiments with data from a real scenario in addition to the MATLAB simulation. Experiments were conducted in the Laboratory at Georgia Tech University described in Section 5.1. In these experiments, we mainly explore how second-order KF compares to first-order KF. The experiment results supported by real data are shown in Figure 15 and Figure 16. These results clearly show that second-order Kalman Filter tracks the actual shadow power more accurately than the first-order Kalman Filter.

Multipath is a Non-Gaussian disturbance in the power signal due to Doppler shifts along different signal paths. As mentioned earlier, Figure 1(b) illustrates some of the possible ways in which multipath signal can occur. Since the phase of the arriving paths is changing fast, the received signal amplitude undergoes rapid fluctuations. In order to model these fluctuations, we generated a histogram of the received signal strength in time. The density function formed by this histogram represents the distribution of the fluctuating values of the received signal strength [2]. Figure 17 shows the pdf output of multipath power, shadow power and total power. We found that 


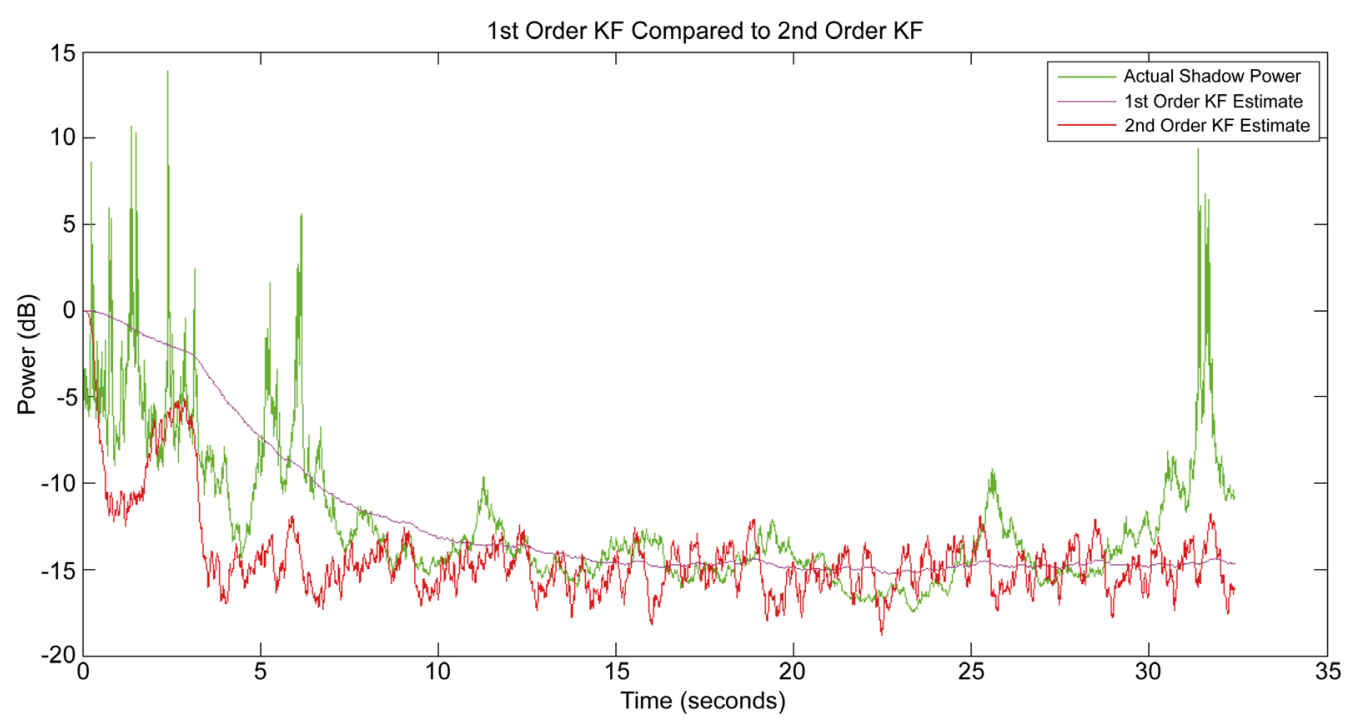

Figure 15. Comparison of first-order KF and second-order KF power estimates using real data.

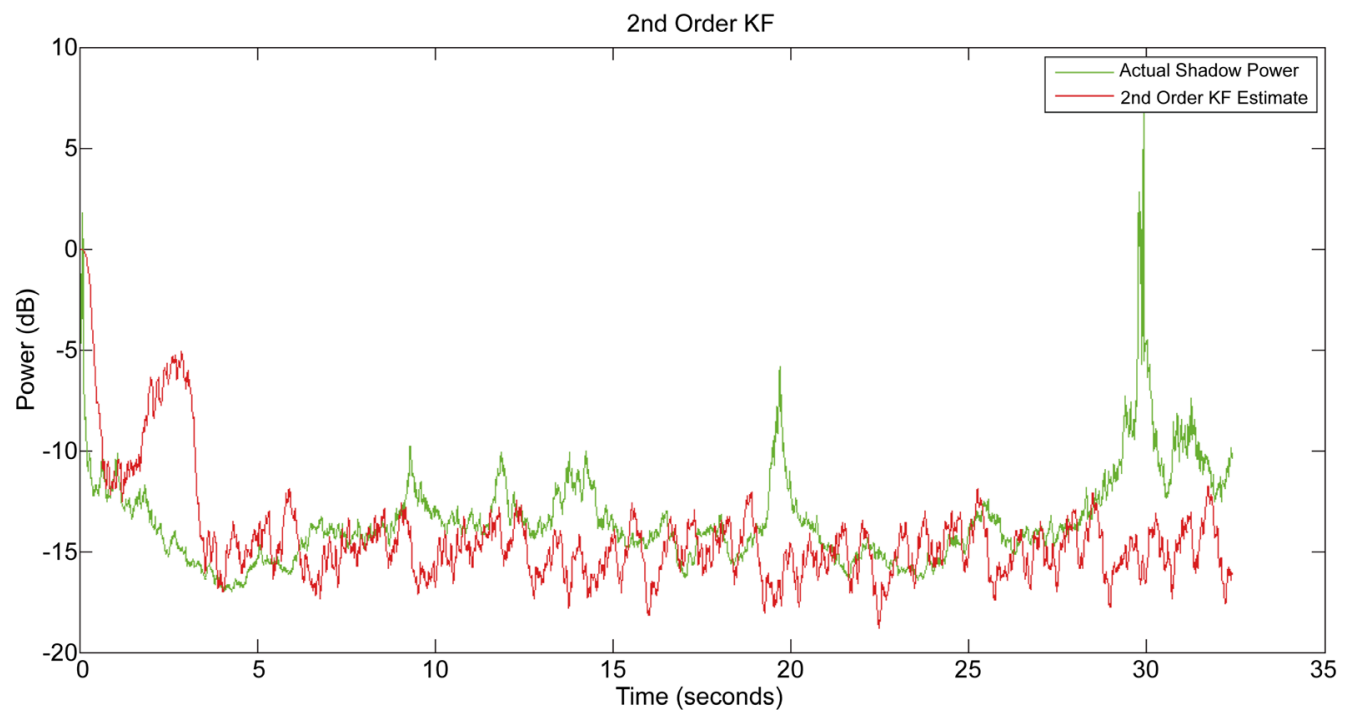

Figure 16. Second-order Kalman Filter applied to real data.

fluctuations due to multipath as well as fluctuations due to shadow power represent Rayleigh distribution. The total power pdf represents a combination of the multipath and shadow outputs.

\section{Conclusions and Future Work}

Our contribution in this paper was to produce new results with second-order state space Kalman Filter to estimate local mean shadow power in mobile communications corrupted by multipath noise. At first, we generated a code to run simulations in order to evaluate the effectiveness of the proposed method by comparing an actual shadow power signal to estimate performance with the linear second-order Kalman Filtering method. Recently, we were able to validate this concept through experiments with an unknown fading model instead of relying on MATLAB simulation results only. Experiments with the real scenario data were conducted to compare the performance of the system with the integrated KF versus the same system without Kalman Filtering. Also, the performances of both the first order state space and the second order state space KF were compared. Experiment results show that the second-order Kalman Filter tracks the actual shadow power more accurately than the firstorder Kalman Filter. Estimation of shadow power was good even when we varied parameters, such as effective 


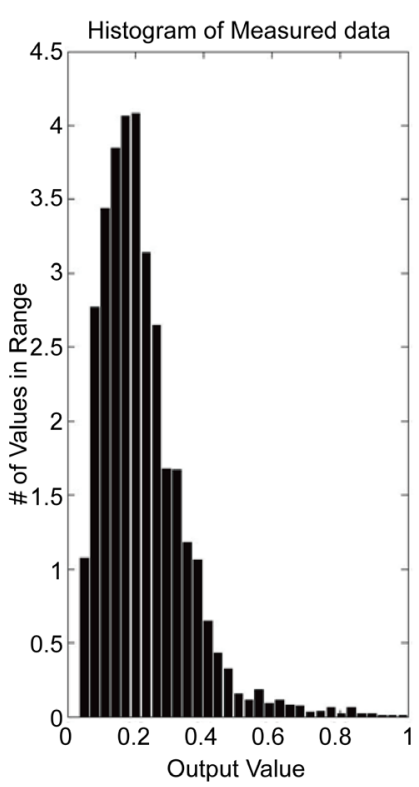

(a)

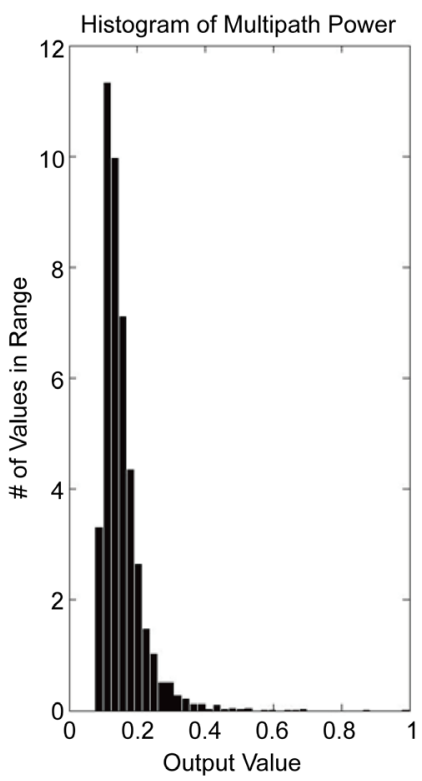

(b)

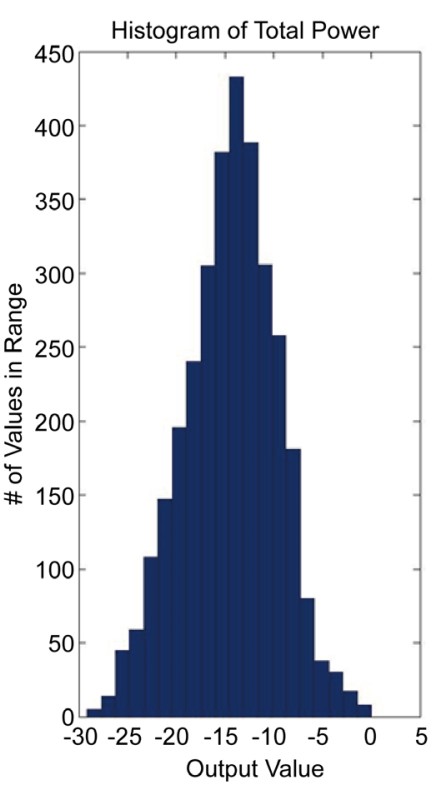

(c)

Figure 17. (a) The pdf output of shadow power, (b) the pdf output of multipath power, and (c) the pdf output of total power.

correlation distance, the number of paths used in the multipath or mobile receiver velocity. The second-order Kalman Filter output has less lag from the actual shadow power.

We also proposed a new adaptive handoff based on a balance of shadow signal strength and quality. Further study of this filter mode is being investigated together with nonlinear channels with imposed non Gaussian noise. We believe that there are opportunities for extending the scope of this study to multi-user and handoff in larger spaces such as parking lots or parks. For example, if there is more than one user in an area, we can create a cluster. The idea is to average roadside signals and clustering road signals as if they are one user.

\section{Acknowledgements}

The authors would like to express profound gratitude to Erica Yaharmatter from Autoliv Inc. for his initial contribution and thought on this subject. They would further like to thank Georgia Tech University for allowing them to use their laboratory facility. Comments and suggestions from two anonymous reviewers on an earlier version of the manuscript were also helpful in improving the presentation of this work.

\section{Conflict of Interest}

The authors declare that they have no conflict of interest.

\section{References}

[1] Jiang, T., Sidiropoulos, N.D. and Giannakis G.B. (2003) Kalman Filtering for Power Estimation in Mobile Communications. IEEE Transactions on Wireless Communications, 2,151-161. http://dx.doi.org/10.1109/TWC.2002.806386

[2] Pahlavan, K. and Krishnamurthy, P. (2002) Characteristics of the Wireless Networks. Prentice Hall PTR, Upper Saddle River. http://www.cwins.wpi.edu/publications/pown/chapter_2.pdf

[3] Patwari, N. (2011) Wireless Communication Systems Course. http://www.ece.utah.edu/ npatwari/pubs/lectureAll_ece5325_6325_f11.pdf

[4] Rappaport, T.S. (2010) Wireless Communications Principles and Practice. 2nd Edition, Persons Education, New York City.

[5] (2016) CDMA-Fading. http://www.tutorialspoint.com/cdma/cdma_fading

[6] (2016) Handoff. https://www.techopedia.com/definition/16851/handoff 
[7] Kalman, R.E. (1960) A New Approach to Linear Filtering and Prediction Problems. Research Institute for Advanced Study, Baltimore.

[8] Simon, D. (2006) Optimal State Estimation: Kalman, $\mathrm{H} \infty$ and Nonlinear Approaches. 1st Edition. John Wiley \& Sons Inc., Hoboken. http://dx.doi.org/10.1002/0470045345

[9] Yarhmatter, E. and Kapetanovic, A. (2012) Power Estimation in Mobile Communications: Comparison of the First Order AR Model to Second Order AR Model. Oakland University, Rochester, Unpublished.

[10] Brown, R.G and Hwang, P.Y.C. (2012) Introduction to Random Signals and Applied Kalman Filtering with Matlab Exercises. 4th Edition, John Wiley \& Sons Inc., Hoboken.

[11] Grewal, M.S. and Andrews, A.P. (2014) Kalman Filtering Theory and Practice Using MATLAB. 4th Edition, John Wiley \& Sons Inc., New York.

[12] Welch, G. and Bishop, G. (2016) An Introduction to the Kalman Filter. University of North Carolina at Chapel Hill, Department of Computer Science, Chapel Hill. http://www.cs.unc.edu/ welch/kalman/

[13] Zeng, Q.A. and Agrawal, D.P. (2010) Handoff in Wireless Mobile Networks. http://cis-linux1.temple.edu/ jiewu/teaching/spring_2010_files/handoff.pdf

[14] Frenkiel, H. (1979) Cellular Radiotelephone System Structured for Flexible Use of Different Cell Sizes. US Patent No. $4,144,411$.

[15] Zetterbeg, P. (2014) Interference Alignment (IA) and Coordinated Multi-Point (CoMP) Overheads and RF Impairments: Test Bed Results. IEEE 80th Vehicle Technology Conference, Vancouver, 14-17 September 2014, 1-7. http://dx.doi.org/10.1109/VTCFall.2014.6966014

[16] Dey, I., Messier, G.G. and Magierowski, S. (2014) Joint Fading and Shadowing Model for Large Office Indoor WLAN Environments. IEEE Transactions on Antennas and Propagation, 62, 2209-2222. http://dx.doi.org/10.1109/TAP.2014.2299818

[17] Mawari, R. and Anderson, A. (2015) Two-Dimensional Small-Scale Fading Modeling and Measurements. Georgia Tech University, Atlanta, Unpublished. 